BNL-112332-2016-JA

File \# 93261

\title{
Fabrication and testing of a newly designed slit system for depth resolved X-ray diffraction measurements
}

John Sinsheimer, Nathalie Bouet, Sanjit Ghose, Eric Dooryhee, Ray Conley

Submitted to Journal of Synchrotron Radiation

November 2016

Photon Sciences Department

Brookhaven National Laboratory

\author{
U.S. Department of Energy \\ USDOE Office of Science (SC), \\ Basic Energy Sciences (BES) (SC-22)
}

\footnotetext{
Notice: This manuscript has been authored by employees of Brookhaven Science Associates, LLC under Contract No. DE- SC0012704 with the U.S. Department of Energy. The publisher by accepting the manuscript for publication acknowledges that the United States Government retains a non-exclusive, paid-up, irrevocable, world-wide license to publish or reproduce the published form of this manuscript, or allow others to do so, for United States Government purposes.
} 


\section{DISCLAIMER}

This report was prepared as an account of work sponsored by an agency of the United States Government. Neither the United States Government nor any agency thereof, nor any of their employees, nor any of their contractors, subcontractors, or their employees, makes any warranty, express or implied, or assumes any legal liability or responsibility for the accuracy, completeness, or any third party's use or the results of such use of any information, apparatus, product, or process disclosed, or represents that its use would not infringe privately owned rights. Reference herein to any specific commercial product, process, or service by trade name, trademark, manufacturer, or otherwise, does not necessarily constitute or imply its endorsement, recommendation, or favoring by the United States Government or any agency thereof or its contractors or subcontractors. The views and opinions of authors expressed herein do not necessarily state or reflect those of the United States Government or any agency thereof. 


\section{Fabrication and testing of a newly designed slit system for depth resolved X-ray diffraction measurements}

John Sinsheimer ${ }^{\mathrm{a}}$, Nathalie Bouet ${ }^{\mathrm{a} *}$, Sanjit Ghose ${ }^{\mathrm{a}}$, Eric Dooryhee ${ }^{\mathrm{a} *}$ and Ray Conley ${ }^{\mathrm{ab}}$

a) National Synchroton Light Source II, Brookhaven National Laboratory, P.O. Box 5000, Upton, NY, 11973-5000, USA

b) Advanced Photon Source, Argonne National Laboratory, 9700 S. Cass Avenue, Lemont, IL, 60439, USA

*Correspondence email: bouet@bnl.gov; edooryhee@bnl.gov

Abstract We have developed a new system of slits called "spiderweb slits" for depth resolved powder or polycrystalline x-ray diffraction measurements. The slits act on diffracted $\mathrm{x}$-rays to select a particular gauge volume of sample, while absorbing diffracted x-rays from outside of this volume. Although the slit geometry is to some extent similar to that of previously developed conical slits or spiral slits, this new design has advantages over the previous ones in use for complex heterogeneous materials and in-situ and operando diffraction measurements. For example, the slits can measure a majority of any diffraction cone for any polycrystalline material, over a continuous range of diffraction angles, and work for X-ray energies of tens to hundreds of kiloelectron volts. The design is generated and optimized using ray-tracing simulations, and fabricated through laser micromachining. The first prototype was successfully tested at the X17A beamline at the National Synchrotron Light Source, and shows similar performance to simulations, demonstrating gauge volume selection for standard powders, for all diffraction peaks over angles of 2 to 10 degrees. A similar, but improved design will be implemented at the X-ray Powder Diffraction beamline at the National Synchrotron Light Source II.

\section{Introduction}

Depth resolved diffraction experiments can be used to nondestructively investigate local structural features or select diffracted x-rays from regions of interest in heterogeneous or multicomponent material systems (Poulsen, 2012). Some examples include using a scanning wire to measure local crystal structure, orientation and strain tensors (Larson et al., 2002), selecting diffracted x-rays with a slit and scanning with an area detector to measure texture (Bunge et al., 2003), or using microchannel plates 
(Wroblewski et al., 1999) or Soller slits (Soller, 1924) to probe local structure. The energy dispersive xray diffraction technique (EDXRD) is used to probe structure of a specific volume of sample by measuring at a specific angle and varying the incident x-ray energy (Buras et. al, 1968; Giessen \& Gordon, 1968), and for example, was recently used to characterize a new, thin battery cathode in-situ and operando (Kirshenbaum et al., 2014). Neutron diffraction with narrow beams and slits can also be used for local stress mapping by scanning through sample regions (Hutchings et al., 2005). Depth resolving techniques are especially important for in-situ experiments that may present unwanted diffraction or scattering sources, such as battery cell containers, reaction cell or pressure cell windows, or for complicated composite structures.

Depth resolved diffraction without scanning can be accomplished through the use of conical slits (Nielsen et. al., 2000) or spiral slits (Martins \& Honkimäki, 2003). Conical slits select diffraction cones from a gauge volume of sample in the Debye-Scherrer diffraction geometry, and unlike other techniques, select nearly the entire diffraction cone from the gauge volume. However, the fabrication of the conical slits limits the design to six predefined diffraction angles for each device. Or a broader range of angles could be measureable by varying the incident $\mathrm{x}$-ray energy. The spiral slit design is a variation of the conical slit, where twelve cut spiral arms can measure any diffraction cone from the selected volume, since the angle of the slit along the spiral arms is varied to match the diffraction angle. However, only twelve small parts of each diffraction cone are imaged on the detector. 

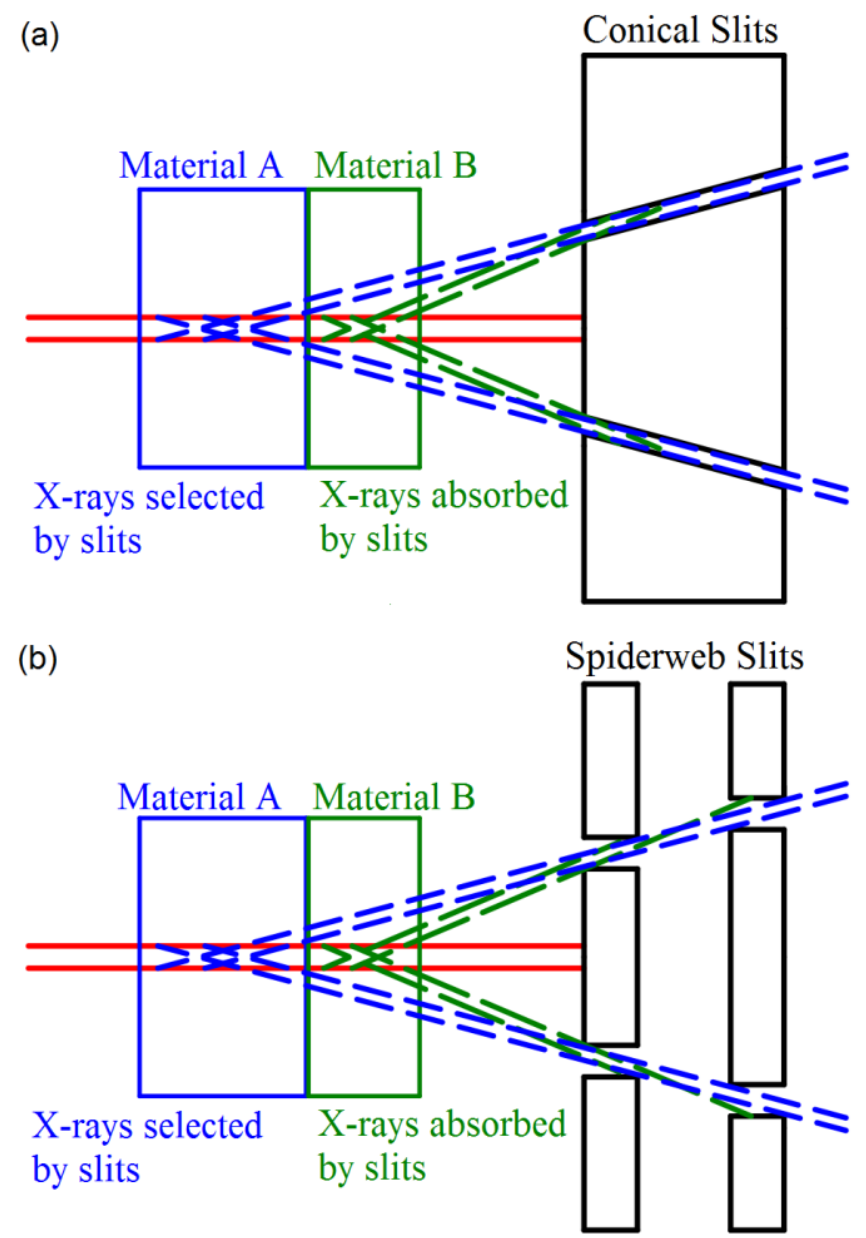

Figure 1 Probing diffraction from a specific gauge volume of sample using (a) conical shaped slit apertures as shown by the blue lines, as in Nielsen et al., 2000. X-rays diffracted from outside the gauge volume are absorbed by the slits as shown by the green lines. (b) The spiderweb slit design functions similarly with two sets of apertures, enabling alternative fabrication techniques and more complex designs.

Conical or spiral slits have been reported to be fabricated using a wire electro-discharge machining (EDM) technique. Wire EDM is well suited to producing thin, deep cuts through dense material. Since diffracted radiation necessarily travels with an angular offset, the ability to cut well defined patterns and at somewhat arbitrary angles makes EDM an attractive fabrication choice in some ways, however some serious limitations appear. Wire EDM requires a starting hole pre-bore and wire feed for every new cut, and the slow feed rate equates to prohibitively expensive fabrication costs if designs with many segments are required. Indeed, conical slits fabricated with wire EDM by others (Nielsen et. al., 2000) were custom tailored both for a particular sample or symmetry group and expected 
diffraction angles. Such customization severely limits the usefulness of the technique to very specific experiments.

We demonstrate a new device enabled by alternative fabrication techniques that maximizes the measured diffraction intensity from a gauge volume of any polycrystalline or powder sample. This device additionally functions for $\mathrm{x}$-ray energies from tens to hundreds of kiloelectron volts and selects a wide range of diffraction angles. Based on its appearance and complexity, we name this newly designed system as "spiderweb slits." The spiderweb slit design consists of two spatially separated stacks of thin absorbing plates as shown in Fig. 1(b), which perform similarly to a conically shaped aperture through a single thick plate as in Fig. 1(a), provided either stack of plates can sufficiently absorb diffracted x-rays. In particular, diffracted $\mathrm{x}$-rays originating from outside the gauge volume that may have a path through either the front or back aperture will be absorbed by the other plate stack, as shown by the green lines in Fig. 1(b). A design involving stacks of thin plates enables many fabrication techniques that can only be applied to thin plates, such as laser micromachining, electroplating, or photolithography combined with reactive ion etching. These fabrication techniques enable far more intricate designs and dimensional freedom than wire-EDM, including such a design with many closely spaced slit apertures.

An important consideration is that any slit acting on diffracted $\mathrm{x}$-rays will select a volume of sample that varies with incident beam size, slit aperture size, and the selected $2 \theta$ diffraction angle. In the powder diffraction geometry considered here, the two dimensional gauge volume selected by the slits has parallelogram shape, as illustrated in Figs. 1 and 3. It is not possible to select exactly the same volume of sample for different $2 \theta$ diffraction angles from slit apertures at other positions. In general, smaller diffraction angles selected by a slit will have larger lengths along the beam path. In this $2 \mathrm{D}$ illustration, the gauge length along the beam direction is given by the equation

$$
\text { Gauge length }=b / \tan (2 \theta)+s / \sin (2 \theta)
$$

where $\mathrm{b}$ is the beam size, and $\mathrm{s}$ is the slit aperture size. A graph of gauge length versus $2 \theta$ diffraction angle for several combinations of beam size and slit aperture size is shown in Fig. 2. For small diffraction angles considered here, the beam size and the slit aperture size are equally important for selecting a limited length of sample along the beam path. The gauge length can be decreased further than shown in Fig. 2 for smaller beam sizes and aperture sizes, but reduces the signal intensity. 


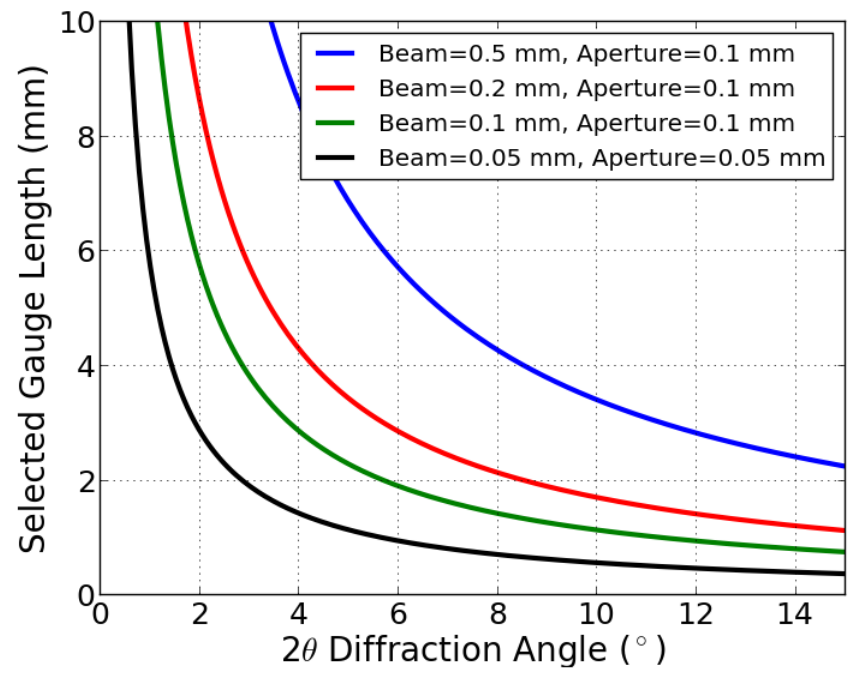

Figure 2 The 2D length of gauge volume selected along the beam direction depends almost equally on the slit aperture size and the beam size, and varies with $2 \theta$ diffraction angle. The gauge length can be further decreased with a smaller beam size or aperture size, but at the cost of reduced signal intensity.

\section{Ray-Tracing Simulations}

Our goal is to develop a design that maximizes the amount of measured diffracted x-rays from the desired sample gauge volume, and so adjacent apertures must be placed as close as possible to one another. However in the described two-plate geometry, if the adjacent apertures are too close to one another or if the plate stacks are too far from one another, certain diffracted x-rays from outside the desired gauge volume may pass through the slit system, causing the design to fail. Possible designs are complex and challenging, given the possible variations in incident beam size, sample size, aperture size, and adjacent aperture separation distances. Therefore, we developed a ray-tracing simulation code written in Python to maximize the density of apertures while ensuring possible diffracted $\mathrm{x}$-rays from outside the gauge volume are absorbed. Other adjustable parameters involved in simulations include the type of material of each plate, the thickness of each stack of plates, the distance between the two plates, and the sample to front plate distance. These parameters are illustrated in Fig. 3. Example values of these parameters used in simulations are given in Table 1. 


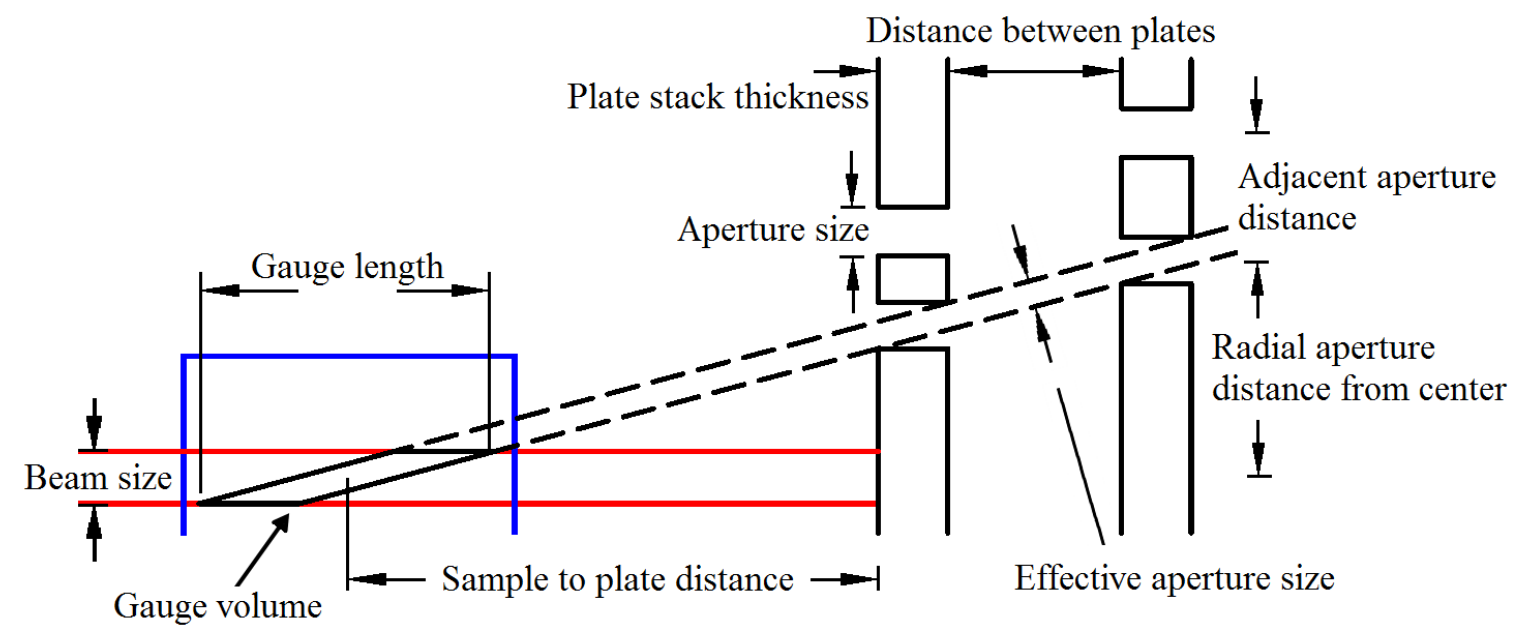

Figure 3 Many parameters affect the shape and size of the probed gauge volume and gauge length in the spiderweb slit system. Simulations with different starting parameters in Table 1 are used to optimize aperture size and adjacent aperture distances.

Table 1 Simulations were performed with multiple combinations of the following parameters in order to optimize aperture size and adjacent aperture distance.

\begin{tabular}{lllll} 
Beam size $(\mathrm{mm})$ & $\begin{array}{l}\text { Sample to plate } \\
\text { distance }(\mathrm{mm})\end{array}$ & $\begin{array}{l}\text { Plate stack } \\
\text { thickness }(\mathrm{mm})\end{array}$ & $\begin{array}{l}\text { Aperture size } \\
(\mathrm{mm})\end{array}$ & $\begin{array}{l}\text { Distance between } \\
\text { plates }(\mathrm{mm})\end{array}$ \\
\hline 0.5 & 50 & 1 & 0.05 & 5 \\
0.2 & 100 & 2 & 0.09 & 10 \\
0.1 & 200 & 3 & 0.20 & 20
\end{tabular}

A number of assumptions are applied to simplify the parameter space and reduce computational time. The geometry is cylindrical and a two dimensional representation is sufficient. The beam divergence is ignored. Since the device was to be tested at the X17A beamline at the National Synchrotron Light Source, we performed simulations matching the beamline characteristics, including a beam size of 200 microns and $\mathrm{x}$-ray energy of $67 \mathrm{keV}$. The simulations assume the incident $\mathrm{x}$-ray beam and possible diffracted $\mathrm{x}$-rays are unattenuated by the sample volume, which is a reasonable simplification at this photon energy. We choose the plate stacks to be made of high purity tungsten for its high x-ray absorption, low cost, and its machinability by laser micromachining or reactive ion etching. We also choose the thickness of each plate stack to be $2 \mathrm{~mm}$, with cut apertures perpendicular through the surface. X-rays originating from outside of the selected gauge volume will then traverse 2 to $4 \mathrm{~mm}$ through tungsten, corresponding to between $2 \mathrm{E}-5$ and 5E-10 x-ray attenuation at $67 \mathrm{keV}$ photon energy. 
Since tungsten has an absorption edge at $69.5 \mathrm{keV}$, the device will function for $\mathrm{x}$-rays up to $120 \mathrm{keV}$ energy with the chosen thickness, and higher energies are possible with a thicker design. The sample is assumed to be 2000 microns thick along the beam path, such that the gauge length will be contained within the sample volume for diffraction angles larger than about 5 degrees. At smaller diffraction angles, the gauge length rapidly becomes larger than the sample thickness as shown in Fig. 2.

Simulations are performed with various values of sample to plate distance, distance between plate stacks, and initial aperture size, as shown in Table 1. To perform the simulation and generate a design, a pair of apertures is first added into the front and back plate stacks to match a particular starting diffraction angle $2 \theta_{1}\left(2 \theta_{1}=2^{\circ}\right.$ in this case $)$ from the center of the sample area, with an initial starting aperture size. We use 90 microns starting aperture size in this case, since this is about the minimum achievable with the chosen laser micromachining process later used for fabrication. This aperture size is equivalent to a 30 micron wide conically shaped aperture, as illustrated by the "effective aperture size" parameter in Fig. 3. Other possible diffracted $\mathrm{x}$-rays at or near $2 \theta_{1}$ from the sample area can also find paths through these apertures. To determine the intensity of diffracted $x$-rays at or near $2 \theta_{1}$ that could reach the detector, the sample area is first divided into a grid of 10 micron square regions, perpendicular to the beam. For example, for a beam size of 200 microns, and a sample thickness of 2000 microns, the sample is divided into a $20 \times 200$ grid. Then for a given diffraction angle around $2 \theta_{1}$, x-rays are drawn from the center of each square and the attenuation of each $\mathrm{x}$-ray through the apertures is calculated. Each $\mathrm{x}$-ray drawn can traverse the full thickness of each plate stack, or a fraction of material in each plate stack, or air. For each diffraction angle around $2 \theta_{1}$, the summed attenuation of the x-rays from all finite elements is calculated, and this value corresponds to the relative diffraction intensity measured by the detector, compared to diffraction intensity without the spiderweb slits. This process is repeated over a range of diffraction angles around $2 \theta_{\mathrm{i}}$, to determine which possible diffraction angles near $2 \theta_{1}$ would reach the detector and to approximately determine the relative intensity, as shown in Fig. 4. The shape and size of this curve depends on all the previously described adjustable parameters. 
(a)

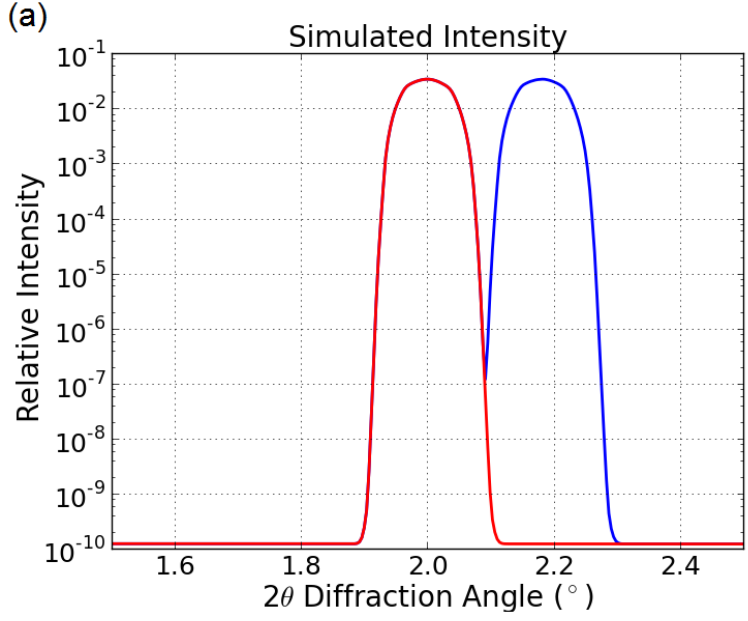

(b)

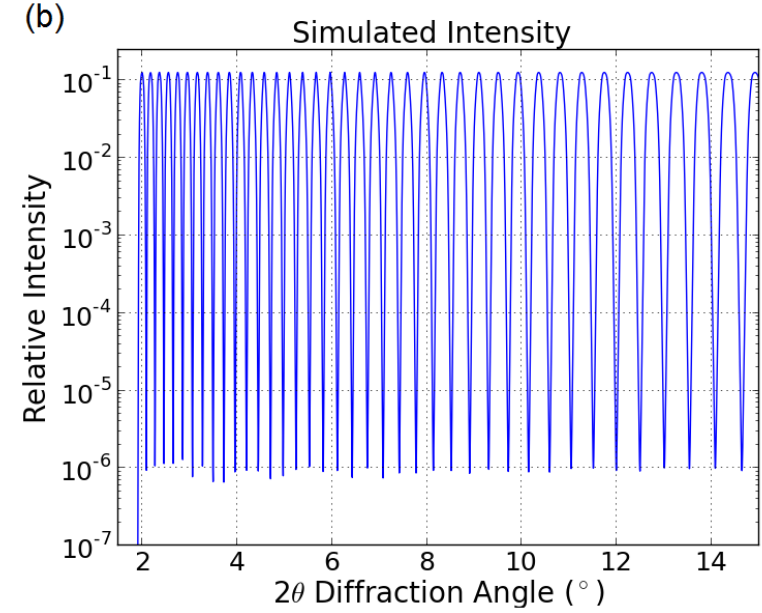

Figure 4 (a) The red curve shows simulated relative intensity passing through a pair of front and back apertures over a range of possible diffraction angles around $2 \theta=2^{\circ}$ from the sample. A second pair of adjacent apertures is added, shown by the blue curve, such that the relative intensity between the peaks reaches a minimum of $10^{-6}$. (b) This process is repeated while optimizing the aperture sizes for $2 \theta$ up to 15 degrees.

The next adjacent pair of apertures is then added with an optimization process to accept x-rays around $2 \theta_{2}$. The distance to the adjacent aperture is considered minimized when the attenuation at a diffraction angle between $2 \theta_{1}$ and $2 \theta_{2}$ reaches an attenuation of $1 \mathrm{E}-6$ or less, corresponding to full attenuation of all the diffracted x-rays through at least one of the two stacks of tungsten plates. The adjacent apertures yield a similar peak shape, and so the width of the first curve between the attenuation value of 1E-6 on either side should be about the same width for the next set of apertures. This approximation is used as a starting point for the next $2 \theta_{i}$ peak center and corresponding aperture coordinates, and small adjustments are made iteratively to maintain 1E-6 attenuation between peaks. In addition, the aperture size is slightly increased to keep the same maximum relative intensity for the next peak. Increasing the aperture size with increasing angle also keeps the gauge lengths more constant as $2 \theta_{i}$ increases. This process of adding a next set of apertures and increasing the aperture size is repeated for diffraction angles $2 \theta_{\mathrm{i}}$ up to 15 degrees, with a completed simulation shown in Fig. 4(b).

A number of possible designs were created and simulated with values of sample to plate distance, distance between plates, and initial aperture size, as in Table 1. Sample to plate distance was simulated in the range of $20 \mathrm{~mm}$ to $200 \mathrm{~mm}$, the distance between plates was simulated between $5 \mathrm{~mm}$ and $30 \mathrm{~mm}$, and initial aperture size was simulated in the range of 50-200 microns. The sample to plate distance determines the density of slit apertures per $2 \theta$ diffraction angle. Closer to the sample, diffraction cones are more densely packed, but adjacent slit apertures require a minimum amount material between them to 
maintain a single selected gauge volume. At larger distances from the sample, the density of slit apertures per $2 \theta$ diffraction angle can be increased, but the plate size must also increase to cover same angular range, and plate size may be limited by the fabrication technique. The distance between the two stacks of plates affects the angular resolution, with larger distances improving the resolution of a particular selected diffraction angle. However, if this distance is too large and slit apertures are closely packed, possible diffracted $\mathrm{x}$-rays from outside the gauge volume may have alternate paths through other combinations of slit apertures and reach the detector. The initial aperture size impacts the gauge length and gauge volume selected by the slits, and the maximum density of slit apertures, and may be limited by the fabrication technique employed. Smaller aperture sizes also require more precise alignment between front and back plates. After performing numerous simulations of combinations of these parameters, the first prototype was selected to have a $100 \mathrm{~mm}$ sample to plate distance, a $20 \mathrm{~mm}$ distance between plate stacks, and a 90 micron starting aperture, with simulation results shown in Fig. 4, for a beam size of 200 microns. These parameters allow for $50 \mathrm{~mm}$ square tungsten plates to select diffraction angles from 2 to 10 degrees, and with gauge lengths of 1 to $0.5 \mathrm{~mm}$ from 5 to 10 degrees. Any x-rays originating from outside the selected gauge volume but along the beam path, were shown to have no paths through the slits for distances up to $30 \mathrm{~mm}$ from the gauge volume.

With one set of densely packed slit apertures, about one third of possible diffraction cones could be measured in this design. Instead of a single set of apertures, it is possible to divide the area of a plate into three sections, with sections offset from one another as shown in Fig. 5(a). Then, any diffraction cone could be measured through at least one of these three azimuthal pie sections. The radial positions and slit aperture widths of these new sections are created by a linear interpolation of the previous set of aperture coordinates, between adjacent apertures, at one third and two thirds the distances along this interpolation. Each of the three sections is simulated as before, with the results and the sum of the three sections shown in Fig. 5(b), shown here over a small range of $2 \theta$. The simulations demonstrate that any diffraction cone should be measurable with this design, with less than $10 \%$ fluctuation of relative peak intensity when averaged over the three sections. 

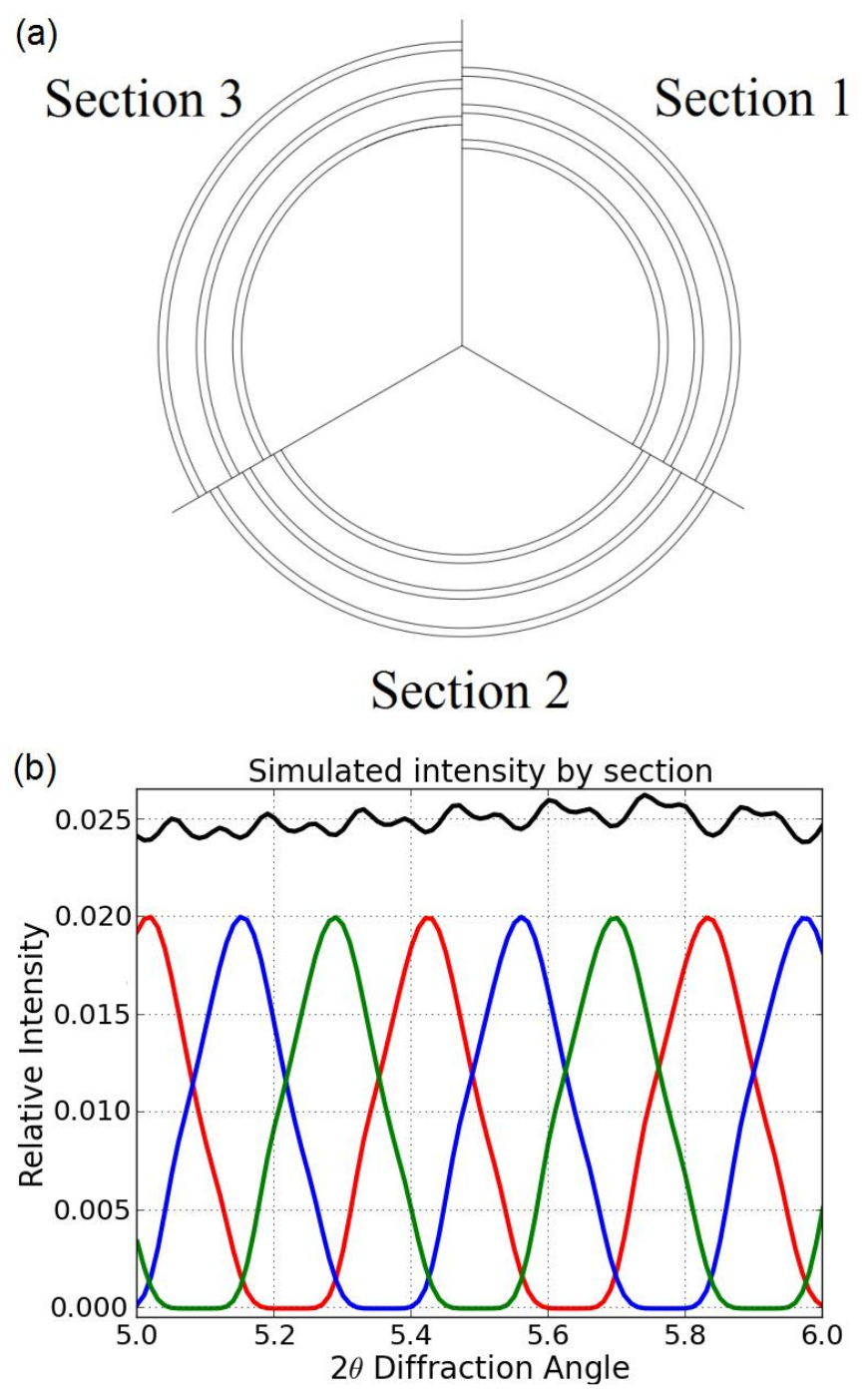

Figure 5 (a) The initial simulation is used to generate two additional offset sections of apertures. (b) These 3 sections are simulated as shown by the red, blue, and green curves, and their sum shown in the black curve, demonstrating that any diffraction angle is measurable in at least 1 of the 3 sections, and the relative intensity between different diffraction peaks varies less than $10 \%$.

\section{Fabrication and Setup}

The optimized simulated slit design coordinates were then used to draw a two dimensional slit design using CAD into a 50 x $50 \mathrm{~mm}$ square area. For the front and back designs, each of three sets of coordinates was drawn into 120 degree sections, with $0.5 \mathrm{~mm}$ gaps between sections. Each of these sections were further divided into four parts by adding back material, with $0.25 \mathrm{~mm}$ gaps extending radially from the center every 30 degrees, to provide structural support to the thin plates. Patterns were cut into a total of sixteen plates each 250 microns thick, forming a $2 \mathrm{~mm}$ thick front stack and $2 \mathrm{~mm}$ thick 
back stack. Five holes of $2 \mathrm{~mm}$ diameter were placed into the design, in the center and near the four corners, for slip fit dowel pins, in order to provide alignment between the front and back stacks of slit plates.

Fabrication was demonstrated with photolithography and reactive ion etching with 100 micron thick tungsten foils at the Center for Functional Nanomaterials at Brookhaven National Laboratory, however the first complete spiderweb prototype was fabricated by laser micromachining, which could be performed more rapidly and with 250 micron thick tungsten foils. The laser micromachining process used was limited to feature sizes of 150 micron for this plate thickness, greater than the previously assumed value of 90 microns used in simulations. To compensate for this limitation, each slit design for the front and back plate stacks are divided into two new designs, where the innermost slit apertures are radially staggered opposite from one another, as shown in Fig. 6, thus creating an effectively smaller slit aperture. This staggered geometry as well as a 6 degree taper angle from the laser micromachining process were simulated and the aperture sizes were further adjusted to yield the previous simulated relative intensities as shown in Figs. 4 and 5(b). A completed etched slit design for a front slit plate is shown in Fig. 7(a). Other fabrication techniques can also be applied, including micro-EDM or lithography and electroplating.

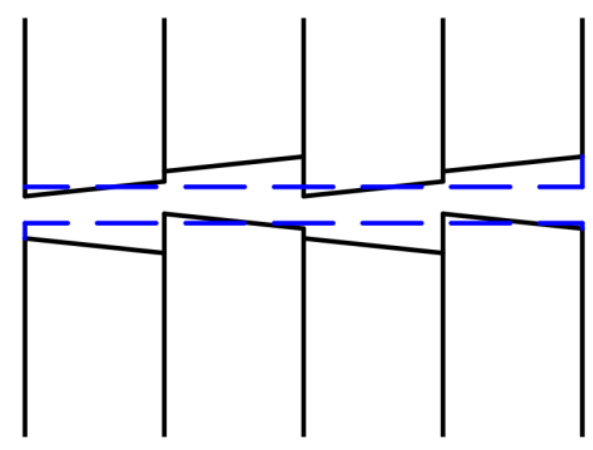

Figure 6 A cross section of the stacked, staggered slit apertures with a 6 degree laser-cut taper angle. The blue dashed line shows the approximate aperture size. 
(a)

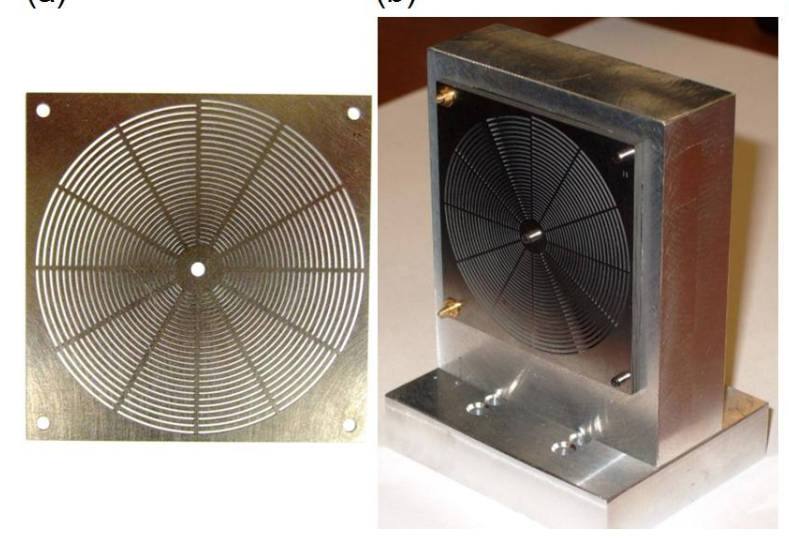

(c)

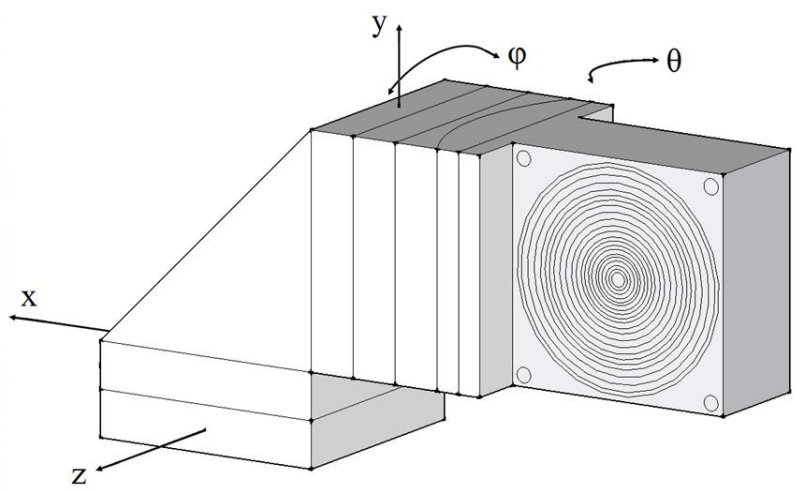

Figure 7 (a) A completed laser-cut spiderweb slit plate. (b) Sixteen plates are mounted on an aluminum holder and aligned with dowel pins. (c) The holder is mounted onto 3 translation stages, a rotation stage, and a mini goniometer, in order to properly align into the diffracted x-rays.

An aluminum mounting was fabricated to provide the $20 \mathrm{~mm}$ separation distance between the front and back slits and to hold the dowel pins aligning the front and back plates. Threaded rods and nuts were used in two of the five holes to help keep the plate stack flush against the holder. This mounting was then attached to a stack of 3 motorized translation stages and 2 rotation stages, in order to align the slits in the diffracted beam as illustrated in Fig. 7(c). The linear actuators had a resolution of 5 microns while the rotation stages had a resolution of about 0.1 degrees. Simulations suggested the alignment in the diffracted beam would need to be within 50 microns translation and 0.2 degrees rotation along both rotational axes.

Alignment was performed using the incident beam and the edges of the central $2 \mathrm{~mm}$ diameter hole. The central alignment rod was removed, and a photodiode was placed behind the opening. By translating horizontally and vertically, perpendicular to the incident beam, the points at which the tungsten began blocking the direct beam could be accurately determined, and then used to center the slits in the beam within 20 microns. The edges of the pair of central holes were also used to correct for rotational offsets within 0.5 degrees.

\section{Results and Discussion}

The performance of the spiderweb slit prototype was assessed at the X17A beamline at the National Synchrotron Light Source. NIST reference powders of nickel and cerium dioxide were used to calibrate the sample to detector distance. The beam size was $0.5 \times 0.5 \mathrm{~mm}$, and the $\mathrm{x}$-ray wavelength (energy) was $0.1839 \AA$ ( $67.4 \mathrm{keV})$. This is a larger beam size than the $0.2 \mathrm{~mm}$ used in the simulations and was unfortunately not able to be reduced further for this experimental run. A $41 \mathrm{x} 41 \mathrm{~cm}$ amorphous 
silicon Perkin Elmer area detector was used to measure diffraction cones with 60 second exposures, and the software program FIT2D (Hammersley, 1998) was used to reduce the two dimensional data into one dimension.

After detector offsets with reference powders were measured, the slits were mounted, aligned, and then tested with the same samples. Each reference powder was approximately $0.5 \mathrm{~mm}$ thick along the beam direction, and was encased in Kapton tape. Fig. 8(a) shows the comparison between measurement of a nickel powder with and without the slits. The 2D detector image in Fig. 8(c) shows that each diffraction cone travels through one of the three sections of slit apertures with high intensity, and through a second adjacent section with much weaker intensity. Importantly, the background scattering at low angles is shown to be significantly reduced by the slits. Since the slits select a much smaller volume of sample than is illuminated by the x-ray beam, the measured diffraction intensity expectedly is much lower. For that reason, the intensity of the diffraction through the slits is attenuated by a factor of $\sim 500$, which is much greater than the factor of $\sim 40$ predicted by simulations. The numerical values of the peak intensities are given in Table 2. All diffraction peaks from the nickel powder are able to be measured over the angular range of 2 degrees to 10.5 degrees, preserving the peak position or $\mathrm{d}$ spacing to within $\pm 0.4 \%$. The relative peak intensities through the spiderweb slits vary $\pm 20 \%$ compared to the reference measurement, larger than the $\pm 10 \%$ variation expected from ray-tracing simulations. The correlation coefficient between these values in Table 2 is 0.99 for the intensity values and greater than 0.9999 for the peak position. The intensity R-factor between the intensities, as determined by the equation 2 , is $17 \%$ :

$$
R_{I}=\sum_{h k l}\left|I_{h k l, o b s}-I_{h k l, c a l c}\right| / \sum_{h k l} I_{h k l, o b s} \times 100
$$

(a)

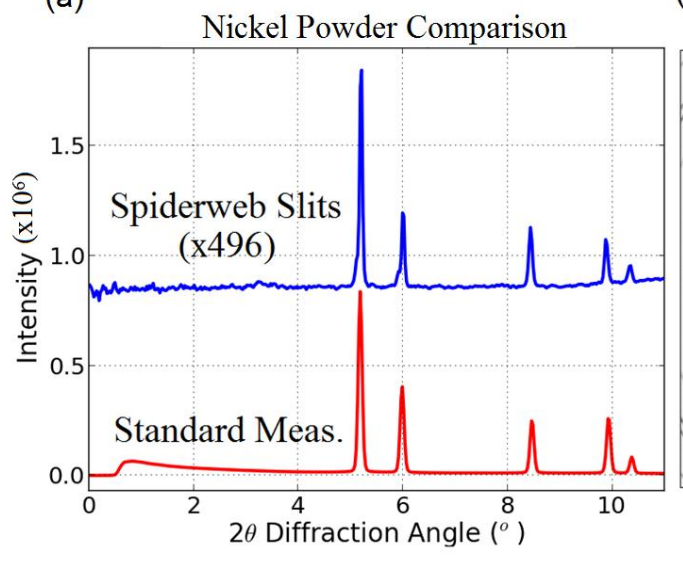

(b)

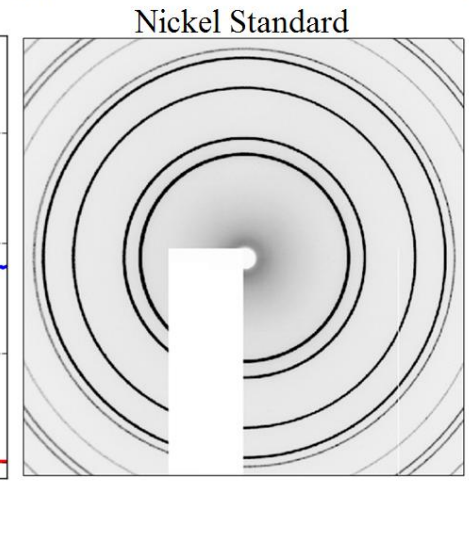

(c) Nickel Standard Through Slits 
Figure 8 (a) A measurement of a standard nickel powder with and without the spiderweb slits. The intensity is scaled by a factor of $~ 500$ to compensate for the reduced scattering volume of sample probed by the slits. (b) A detector image of the raw data before the slits are inserted. (c) A detector image with the slits shows at least $1 / 3$ of each diffraction cone from the powder. A rectangular mask is used due to a faulty detector panel.

Table 2 A comparison of the background-subtracted peak intensities and calculated d spacings of the nickel reference powder shown in Fig. 8.

\begin{tabular}{c|cccccc}
\multicolumn{3}{l}{ Intensity (counts) } & \multicolumn{7}{c}{$\mathrm{d}(\AA)$} \\
$\mathrm{hkl}$ & Reference & Slits In*496 & \% Difference & Reference & Slits In & \% Difference \\
\hline 111 & $8.23 \mathrm{E}+05$ & $9.87 \mathrm{E}+05$ & 20 & 4.069 & 4.052 & 0.41 \\
200 & $3.96 \mathrm{E}+05$ & $3.32 \mathrm{E}+05$ & -16 & 3.523 & 3.510 & 0.37 \\
220 & $2.38 \mathrm{E}+05$ & $2.66 \mathrm{E}+05$ & 12 & 2.492 & 2.497 & -0.23 \\
311 & $2.48 \mathrm{E}+05$ & $2.01 \mathrm{E}+05$ & -19 & 2.125 & 2.133 & -0.37 \\
222 & $7.50 \mathrm{E}+04$ & $7.74 \mathrm{E}+04$ & 3 & 2.035 & 2.039 & -0.19
\end{tabular}

Next, the gauge length and gauge volume selected by the spiderweb slits were measured by translating the gauge volume away from the thin nickel powder along the incident beam axis. As the selected gauge volume moves out of the sample volume, we expect the diffraction peak intensity to be increasingly attenuated. The linear actuator translated the slits along the beam direction, denoted as ' $\mathrm{z}$ ' here, with small corrections to $\mathrm{x}$ and $\mathrm{y}$ directions due to misalignment of the $\mathrm{z}$ motor along the beam direction. Measurements were performed at various distances, shown in Fig. 9. After translating the slits $6 \mathrm{~mm}$ off center from the nickel powder, the diffracted x-rays above 6 degrees are completely absorbed, and the first two diffraction peaks are attenuated by a factor of about 10 . These results are consistent with the blue curve in Fig. 2, where the gauge length is $6 \mathrm{~mm}$ at a diffraction angle of 6 degrees for a beam size of $0.5 \mathrm{~mm}$ and aperture size of $0.1 \mathrm{~mm}$. The large gauge length here depends more on the larger beam size of $0.5 \mathrm{~mm}$ than the $0.1 \mathrm{~mm}$ effective aperture size, and could be easily reduced with a smaller incident beam. 


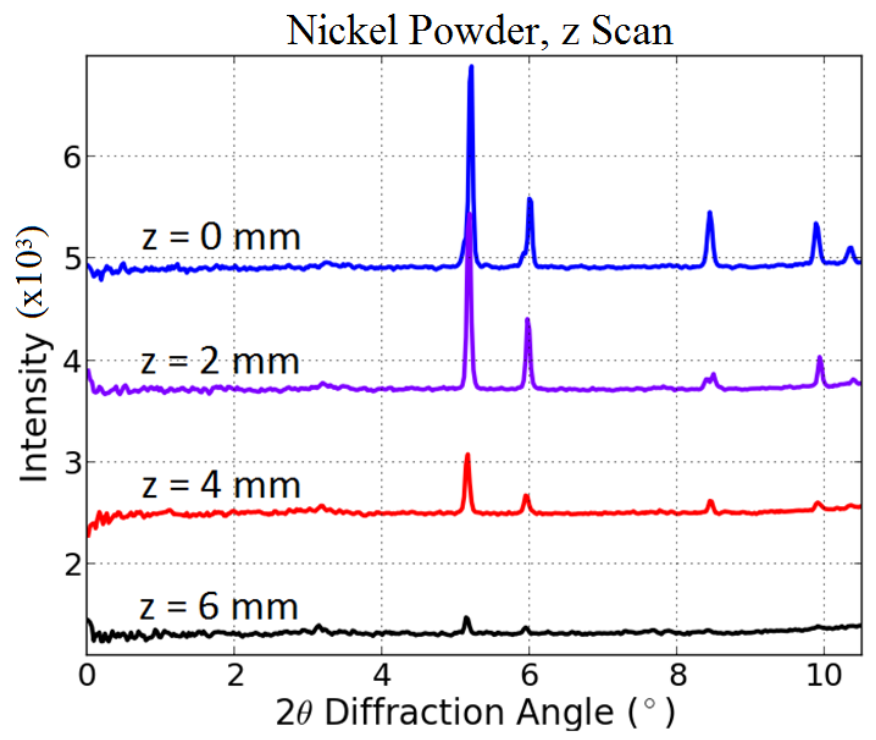

Figure 9 The slits are translated along the beam direction, $\mathrm{z}$, moving the gauge volume out of the diffracting sample volume. Diffracted x-rays from outside the selected gauge volume are rapidly attenuated, especially at higher diffraction angles.

The previous experiments were repeated with a reference ceria powder. Fig. 10 shows the comparison between the slits-out measurement and the slits-in measurement (i.e. using the spiderweb slits). The absorption is much greater for the low diffraction angles, through the staggered apertures as in Fig. 6, compared with all other diffraction angles. This suggests that the slits are more effective for small-d structures whenever the intensity of the scattering contributing to the lowest angles is less. This is probably caused by the large cutting roughness of the laser micromachining process, around 20 microns, compared to the small effective conical aperture size of 50 microns. Additionally, if the tungsten plates are not flat against the holder or bowing outward, the effective thickness of the stack will be greater, reducing the effective aperture size. This effect is more pronounced for the smallest apertures. Above 4 degrees, similar results are observed when compared to the nickel powder measurement, and a best scaling factor of 400 is calculated to match the peak intensities over this range. However a much larger fluctuation of relative peak intensities, up to $\pm 37 \%$, through the slits is observed compared to the reference measurement. Again all diffraction peaks are measureable and the peak positions are well preserved to within $\pm 0.4 \%$ of the reference. The correlation coefficient between the two values are 0.96 for the intensities, greater than 0.9999 for the peak positions, and the intensity R-factor is $25 \%$, excluding the first two peaks for these calculations. 


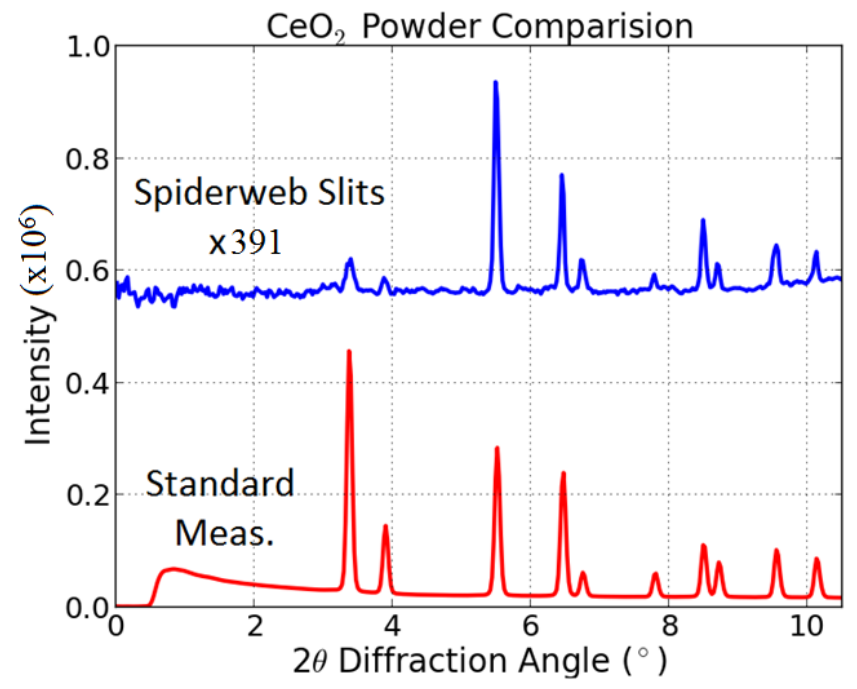

Figure 10 A measurement of a ceria reference powder with and without the spiderweb slits. The curve through the slits is scaled by $\sim 400$ to best match the relative peak intensities of the reference.

Table 3 A comparison of the background-subtracted diffraction peak intensities and calculated d spacings for the ceria reference powder of Fig. 10.

\begin{tabular}{|c|c|c|c|c|c|c|}
\hline \multirow[b]{2}{*}{ hkl } & \multicolumn{3}{|c|}{ Intensity (counts) } & \multicolumn{3}{|l|}{$\mathrm{d}(\AA)$} \\
\hline & Reference & Slits In*391 & $\%$ Difference & Reference & Slits In & $\%$ Difference \\
\hline 111 & $4.25 \mathrm{E}+05$ & $8.02 \mathrm{E}+04$ & -88.1 & 6.243 & 6.219 & 0.38 \\
\hline 200 & $1.20 \mathrm{E}+05$ & $3.79 \mathrm{E}+04$ & -80.0 & 5.410 & 5.425 & -0.29 \\
\hline 220 & $2.62 \mathrm{E}+05$ & $3.42 \mathrm{E}+05$ & 30.3 & 3.824 & 3.828 & -0.10 \\
\hline 311 & $2.18 \mathrm{E}+05$ & $1.85 \mathrm{E}+05$ & -15.0 & 3.261 & 3.263 & -0.06 \\
\hline 222 & $4.15 \mathrm{E}+04$ & $5.00 \mathrm{E}+04$ & 20.5 & 3.123 & 3.122 & 0.03 \\
\hline 400 & $4.14 \mathrm{E}+04$ & $2.59 \mathrm{E}+04$ & -37.4 & 2.706 & 2.707 & -0.04 \\
\hline 331 & $9.17 \mathrm{E}+04$ & $1.14 \mathrm{E}+05$ & 24.5 & 2.482 & 2.481 & 0.07 \\
\hline 420 & $6.10 \mathrm{E}+04$ & $4.22 \mathrm{E}+04$ & -30.9 & 2.420 & 2.421 & -0.02 \\
\hline 422 & $8.40 \mathrm{E}+04$ & $6.36 \mathrm{E}+04$ & -24.3 & 2.209 & 2.207 & 0.08 \\
\hline 511 & $6.87 \mathrm{E}+04$ & $5.15 \mathrm{E}+04$ & -25.1 & 2.083 & 2.082 & 0.04 \\
\hline
\end{tabular}

To further test the material selection capability, the nickel powder and ceria powder were both placed into the path of the $\mathrm{x}$-ray beam, spaced $6 \mathrm{~mm}$ apart. The slits were first aligned to select 
diffraction from the nickel powder, and then measurements were performed as the slits were translated toward the ceria powder. These results are shown in Fig. 11. Fig 11(a) shows three plots: when the slits are aligned with the nickel powder only, at $\mathrm{z}=0 \mathrm{~mm}$, when the slits select the volume between the two samples at $\mathrm{z}=3 \mathrm{~mm}$, and when the slits select diffraction from ceria only at $\mathrm{z}=6 \mathrm{~mm}$. Measurements were performed in steps of $0.5 \mathrm{~mm}$ in $\mathrm{z}$, shown in a $2 \mathrm{D}$ intensity map of $\mathrm{z}$ versus $2 \theta$ diffraction angle in Fig. 11(b). At the largest diffraction angles here, we observe complete attenuation of either ceria at $\mathrm{z}=0$ $\mathrm{mm}$ and nickel at $\mathrm{z}=6 \mathrm{~mm}$, due to the geometrically shorter gauge length along the beam direction, as calculated in Fig. 2. At lower angles and larger gauge lengths, such as 5 degrees, the first nickel diffraction peak still reaches the detector at $\mathrm{z}=6 \mathrm{~mm}$, attenuated by about a factor of 10 , consistent with the results shown in Fig. 9. Note the overall diffraction intensity is lower for the ceria powder due to absorption of the direct beam by the nickel powder and due to the different thicknesses of the powders.

(a)

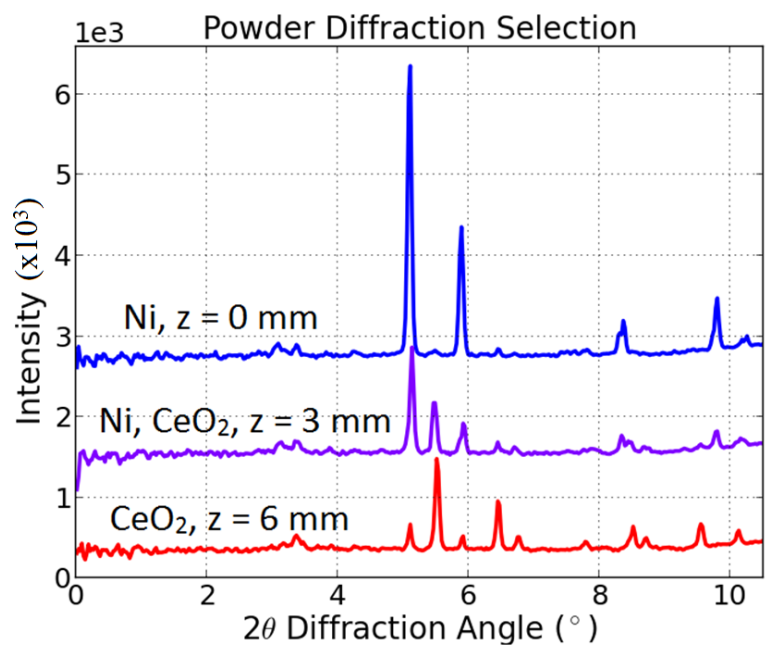

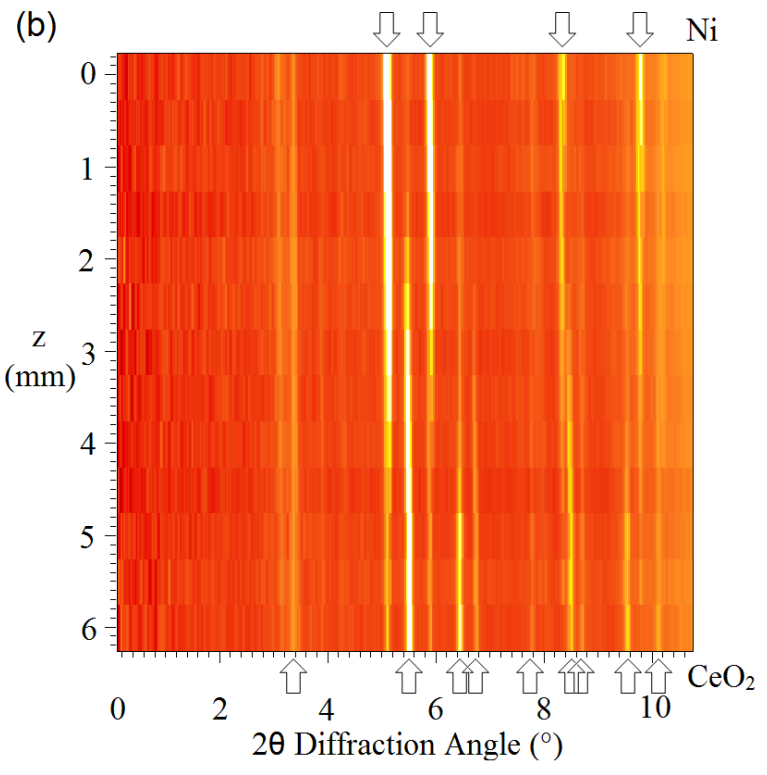

Figure 11 Spiderweb slits are used to select diffraction from either a nickel or ceria reference powder, both in the direct x-ray beam and $6 \mathrm{~mm}$ apart. (a) Diffraction intensities for 3 positions of the spiderweb slits. (b) A top-down view of (a), for a z-scan with $0.5 \mathrm{~mm}$ increment. The nickel selection at $\mathrm{z}=0 \mathrm{~mm}$ is shown on the top of the graph, while ceria selection at $\mathrm{z}=6 \mathrm{~mm}$ is shown on the bottom. Arrows at the top of (b) indicate the diffraction peaks from nickel, while arrows at the bottom indicate diffraction peaks from ceria.

Some deviations from the ray-tracing simulations are expected from misalignment of the slit plate stacks. Slit plate stacks need to be closely aligned to one another on the holder, along the beam direction, and must be aligned into the diffracted beam. Misalignment causes some regions of the spiderweb slits to have smaller effective aperture sizes while opposite regions to have larger effective aperture sizes, 
skewing the relative peak intensities. These effects become more pronounced for the smaller effective apertures, at smaller diffraction angles. Other potential designs that may have smaller effective apertures would require greater control over these alignments. The sidewall roughness of the apertures also will cause a variation in intensities through the apertures. Fabrication was demonstrated with reactive ion etching, which greatly reduces the sidewall roughness compared to laser micromachining, but is a more difficult fabrication process.

A close examination of certain diffraction peaks through the slits show a small shoulder on one side of the peak. This is due to the discontinuous jump from section to section, which was expected to average into a smooth peak in the simulations. This effect is eliminated with a new design that converts the 3 discrete sections into continuously varying sections, in a spiral-like shape. These sections can be readily converted into such a design by linearly interpolating both the radial position and aperture size between sections, and could be refined through simulations.

This improved spiderweb slit system has already begun to be developed, and is expected to become a tool at the X-ray Powder Diffraction beamline at the National Synchrotron Light Source II. The alignment of the slits in the diffracted beam can also be improved by adding in rotational symmetry into the slit design. If instead of single spiral shape, two interwoven spirals are used with 2-fold symmetry, then the alignment can be easily verified with standard powders. Once the slits are properly aligned, the detector will measure two symmetrical parts of each diffraction cone, having 2-fold symmetry. Any misalignment would break this symmetry, showing a large section of a diffraction cone on one side, and a smaller section of a diffraction cone opposite. With this new design, it may become possible to rotate the slit system to capture full diffraction cones, provided the detector integrates counts over half of a revolution. This technique could be used for depth-resolved measurements of texture or strain.

The spiderweb slits are applicable to a variety of use cases. An important example is to study insitu structural changes that occur in the electrode material as a function of depth of charge/discharge for a range of battery systems. The slits provide space resolution to separate out the various components of the battery (outside can or envelope or cover, cathode, electrolyte or separator, anode, current collector, gasket, etc) and provide phase profile. The slits can be used to remove most unwanted signal from materials surrounding the sample inside an environmental cell. We shall use the slits at the NSLS-II XPD beamline to probe the sample in a high pressure, high temperature reactor while minimizing contribution from the upstream and downstream windows. 


\section{Conclusion}

We have developed a new slit system, called spiderweb slits, to provide rapid depth-resolved xray powder diffraction. This design is far more versatile compared to previous methods, since the slits can select most of any diffraction cone from a gauge volume over a continuous range of diffraction angles, without the need for scanning any parts of the system. This capability is especially important for in-situ and operando experiments. The device demonstrated here functions up to $120 \mathrm{keV}$, but this design can be easily extended to function for tens to hundreds of $\mathrm{keV}$ x-ray energies, and larger plates could be fabricated to capture larger diffraction angles. An optimized design via ray-tracing simulations was fabricated through laser micromachining fabrication and tested at the X17A beamline at the National Synchrotron Light Source with standard powders. The experiments demonstrate gauge volume selection consistent with simulations and the ability to measure all diffraction peaks over the design range, while preserving relative diffraction peak intensity within $\pm 37 \%$, and preserving the diffraction peak position to within $\pm 0.4 \%$. An improved design to better preserve relative diffraction peak intensity and peak shape is expected to become a unique tool at the X-ray Powder Diffraction beamline at the National Synchrotron Light Source II.

Acknowledgements The authors would like to thank John Trunk, Bill Smith, and Dennis Kuhn for fabricating the slit mountings, and Jun Ma, Dennis Poshka, and Wayne Lewis for wiring and configuring the slit motors. This work was supported by the Brookhaven National Laboratory Laboratory-Directed Research and Development Program. Use of the National Synchrotron Light Source and the Center for Functional Nanomaterials, Brookhaven National Laboratory, was supported by the U.S. Department of Energy, Office of Science, Office of Basic Energy Sciences, under Contract No. DE-AC02-98CH10886. Laser-micromachining was performed by Potomac Photonics.

\section{References}

Bunge, H.J., Wcislak, L., Klein, H., Garbe, U. \& Schneider, J. R. (2003). J. Appl. Cryst. 36, 1240-1255. Buras, B., Chwaszczewska, S., Szarras, S. \& Szmid, Z. (1968). Inst. Nucl. Res. (Warsaw), Rep. No. 894/II/PS.

Giessen, B.C \& Gordon, G.E. (1968). Science. 159, 973.

Hammersley, A. P. (1998). ESRF Internal Report, ESRF98HA01T, FIT2D V9.129 Reference Manual V3.1.

Hutchings, M. T., Withers, P. J., Holden, T. M., and Lorentzen, T. (2005). Introduction to the Characterization of Residual Stress by Neutron Diffraction. CRC Press. 
Kirshenbaum, K.C., Bock, D.C., Zhong, Z., Marchilok, A.C., Takeuchi, K.J. \& Takeuchi, E.S. (2014).

Phys.Chem.Chem.Phys. 16, 9138.

Larson, B.C., Yang, W., Ice, G. E. , Budai, J. D. \& Tischler, J. Z. (2002). Nature. 415, 887-890.

Martins, R.V. \& Honkimäki, V. (2003). Textures and Microstructures. 35, 3/4, 145-152.

Nielsen, S.F., Wolf, A., Poulsen, H.F., Ohler, M., Lienert, U. \& Owen, R.A. (2000). J. Synchrotron Rad.

7, 103-109.

Poulsen, H. F. (2012). J. Appl. Cryst. 45, 1084-1097.

Soller, W. (1924). Phys. Rev. 24, 158-167.

Wroblewski, T., Clauß, O., Crostack, H.-A., Ertel, A., Fandrich, F., Genzel, Ch., Hradil, K., Ternes, W. \& Woldt, E. (1999). Nuclear Instruments and Methods in Physics Research. A 428, 570-582. 